\title{
Procedure for the steady-state verification of modulation-based noise reduction systems in hearing instruments
}

\author{
Jesko G Lamm*, Anna K Berg, Christian M Künzler, Bernhard Kuenzle and Christian G Glück
}

\begin{abstract}
Hearing instrument verification involves measuring the performance of modulation-based noise reduction systems. The article proposes a systematic procedure for their verification. The procedure has the potential for application in the verification of other signal processing systems, because it is independent of the hearing instrument domain. Its key concept, the separation of abstract and concrete design of test signals, has been adopted from the embedded systems domain. Specifically for modulation-based noise reduction systems in hearing instruments, the article shows a complete implementation of the verification procedure, proposing improvements of existing measurement techniques. To fully cover the verification procedure, a new measurement approach based on maximum length sequences and DFT processing is introduced, revisiting concepts of system identification that came up in the 1970s. These can easily be used with the computational resources of today's microcomputers. Sample measurements with existing hearing instruments demonstrate the verification procedure with different measurement techniques.
\end{abstract}

\section{Introduction}

A hearing instrument should assist its user by amplifying sound with a certain gain, but can also cause discomfort in noisy environments. Therefore, its noise reduction subsystem should reduce the hearing instrument's gain while noise is present - usually dependent on frequency in different subbands. To preserve speech understanding, the noise reduction should avoid gain reduction in those subbands that contain speech. Based on the observation that speech has a characteristic modulation spectrum [1], a modulation-based noise reduction should detect speech by its modulation [2], which is the fluctuation of a subband signal's envelope over time. As a consequence, modulation-based noise reduction will reduce gain more strongly in a subband carrying unmodulated sound than in a subband with modulated sound [3], as it has been illustrated in Figure 1.

This article describes the verification of a noise reduction subsystem within the fully integrated hearing instrument. By verification we mean the confirmation of

\footnotetext{
* Correspondence: jla@bernafon.ch

Bernafon AG, Morgenstrasse 131, 3018 Bern, Switzerland JGL: EURASIP
} Member

\section{勿

compliance with the specified requirements, here, by measurement. This means that the scope of this article is limited to the measurement of system responses rather than a clinical verification of the noise reduction functionality under test.

Measuring system responses with test signals is a typical problem of system identification and has been solved with measurement techniques based on test signals that meet some typical requirements regarding their power spectrum and their amplitude distribution. Particularly, the minimization of peaks has been of interest with regard to the fact that practical systems have a limited dynamic range. However, the synthesis of test signals that allow enforcing a signal feature like modulation has only recently been proposed $[4,5]$.

This article puts the synthesis techniques of prior work into the context of systematic verification, focusing the so-called coverage of the system's input parameters. We show how to systematically design sets of test signals that drive the system under test into a number of different states, allowing to confirm a complete verification of the subsystem of interest.

A process for achieving the systematic verification is needed. While processes targeted at test coverage have 


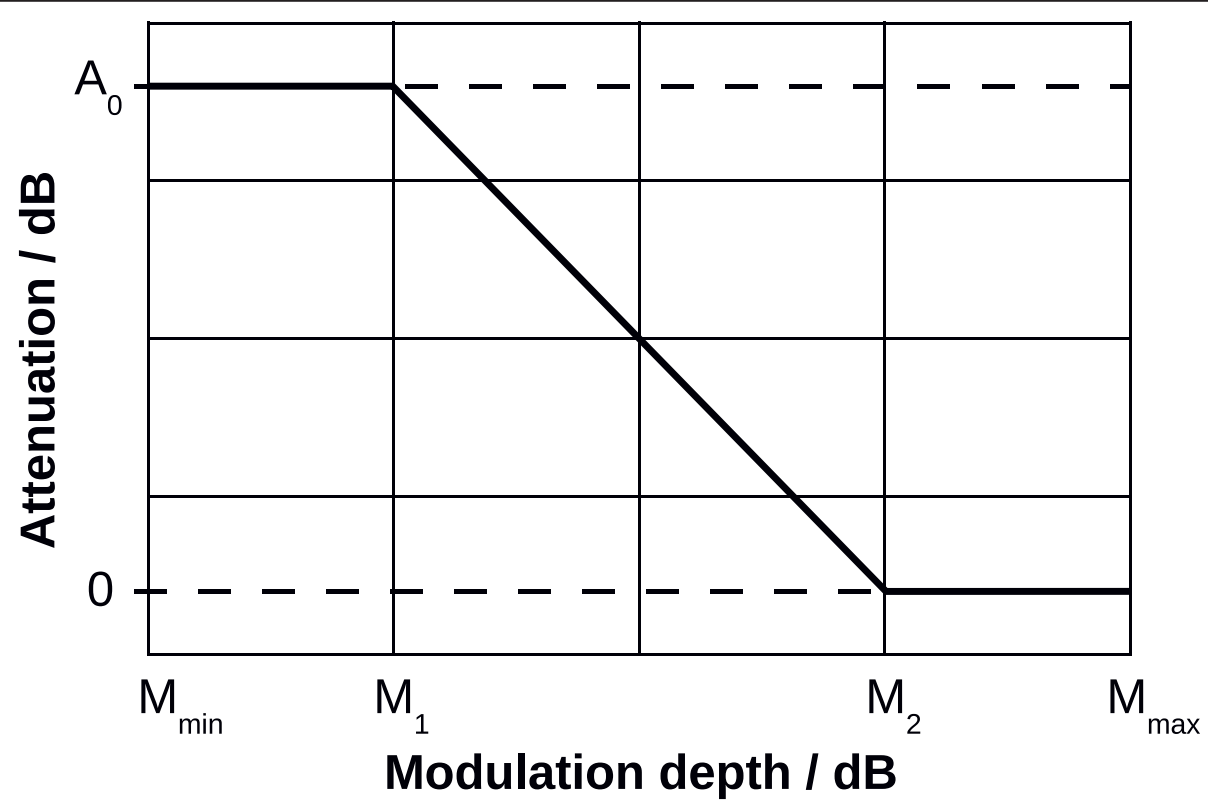

Figure 1 The gain reduction (attenuation) of a modulation-based noise reduction system is strongest for low modulation depth. Figure based on [3].

been described for the verification of purely softwareoriented systems (e.g., [6]) or simple signal processing systems like e.g., control units in automotive technology $([7,8])$, there has not yet been such work for systems that should provide intense digital signal processing, like e.g., noise reduction subsystems in hearing instruments.

This article introduces test design techniques for signal processing systems by combining existing test processes from the embedded systems domain with signal design techniques from system identification into a novel verification procedure. Its key concept is to obtain an abstract description of test sequences first, in order to derive concrete test signals in a second step. Since these will have to be synthetic in order to match the criteria defined by the test sequence, they are not suitable for testing the system under realistic conditions.

It is therefore a prerequisite for the procedure to have requirements toward the system under test stated in a technical, measurable way. A typical application is the regression testing in product development where the performance characteristics of the system under development are re-assessed after implementation changes. Typically, additional tests under realistic conditions (e.g., a clinical trial) are needed before a product can be released, but these are out of scope of the presented procedure.

We believe that the verification procedure is applicable to different kinds of signal processing systems, because one of its essential parts-the abstract description of test signals that can be implemented with different synthesis techniques-is independent of the kind of application, but also because applications other than hearing instruments have to deal with subsystems similar to a noise reduction, e.g., being based on signal features (like e.g., music classification for portable devices [9]) or having to adapt their processing based on information encoded in the signal (like e.g., voice activitydependent transmission systems in telephony [10]). Therefore the procedure itself will be described independently of our application area, hearing instruments.

After introducing definitions of terms and concepts as well as the proposed verification procedure, the article will report experiments demonstrating the procedure with modulation-based noise reduction subsystems of hearing instruments. In contrast to previously reported measurements for verifying these $[4,5,11,12]$, the ones presented here are based on a design for test coverage that is derived from the requirements toward the subsystem under test.

\section{Definitions}

\subsection{Definitions related to signal processing 2.1.1 Signals}

This section defines different kinds of signals to be used in measurements.

- A signal whose amplitude has only two discrete values is called a binary signal.

- A perfect sequence is a stimulus whose spectral components are constant over the whole Nyquist 
frequency range (see e.g., [13] for a more formal definition of a perfect sequence).

\subsubsection{Frequency response measurements}

This section defines different ways of measuring frequency responses of the system or one of its subsystems. They are based on digital signal processing and thus assume that test stimuli and the output signal of the system under test are available as digital waveforms, as shown in Figure 2 where one digital waveform $x$ enters the system under test and another one, $y$, is its output signal. For systems with analog transducers like hearing instruments, the signals $x$ and $y$ have to be interfaced to the system under test via a digital-to-analog and an analog-to-digital converter. This is omitted for simplicity in Figure 2.

NLMS-based measurement As an improvement of the least mean squares (LMS) algorithm that has been introduced by Widrow and Hoff [14], Nagumo and Noda [15] have introduced the normalized least mean squares (NLMS) algorithm. It iteratively approximates the impulse response $\mathbf{h}(\mathrm{n})$ of the system under test with tap weight factors $\hat{\mathbf{h}}(\mathrm{n})$ of an adaptive filter whose frequency response can be used as an estimate of the system's frequency response $\mathrm{H}(f)$.

DFT-based measurements Processing the signals $x$ and $y$ with the Discrete Fourier Transform (DFT) can approximate the frequency response function $H(f)$ of the system $h(n)[16,17]$. For the specification of the detailed computation, let the frequency bin number $k$ of the DFT of one frame of signal $x$ at discrete time $n$ be $X_{n}$ $(k)$. Let $Y_{n}(k)$ be the according value of signal $y$. Then, the corresponding frequency bin $H_{n}(k)$ of the approximated frequency response is given by the equation below.

$$
H_{n}(k)=\frac{Y_{n}(k)}{X_{n}(k)}
$$

Differential measurements Observing the frequency response of one of a linear system's subsystems is possible by differential measurements [4], i.e., a combination of two separate measurements with an identical stimulus, once with the subsystem of interest activated and once having it deactivated. Dividing the frequency responses of both measurements can show the effect of the subsystem of interest on the frequency response of the whole system.

\subsubsection{Modulation/modulation frequency}

The modulation definition from the introduction was based on the signal envelope, which is often used in quantifying modulation (e.g., as the basis of the modulation spectrum like in [1]) and shall therefore also be used here as a basis of a first definition related to modulation: from the observation of signal envelope within one time frame let the observed minimum be the modulation valley, and let the maximum be the modulation peak accordingly. We use $\mathrm{m} \in\left[M_{\min }, M_{\max }\right]$ to denote

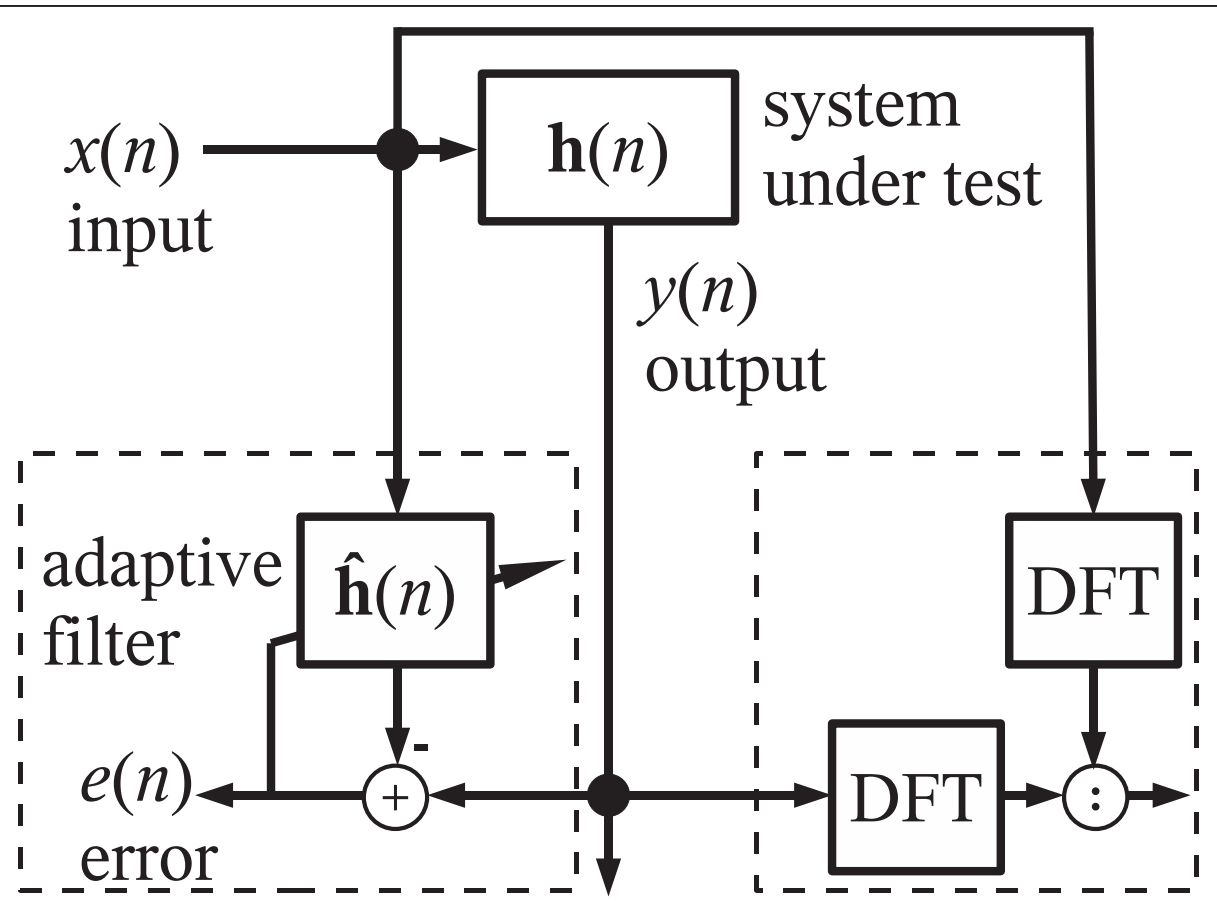

Figure 2 Definition of operators and signals involved in frequency response approximation. 
the modulation depth and define:

$$
m / \mathrm{dB}=10 \cdot \log _{10} \frac{\text { signal power at modulation peaks }}{\text { signal power at modulation valleys }} .
$$

This definition is a good basis for developing hearing instruments and is also valid for the samples used in the experimental part of this article; however, it is undefined if the denominator becomes zero, it relates to only one time frame and is furthermore dependent on time constants of envelope estimators and power estimators, which are usually not specified in the data sheet of a hearing instrument. For the construction of synthetic stimuli, we therefore propose another approach for defining the modulation depth: for a given subband with index $b$, we define the corresponding modulation depth $m_{b}$ indirectly, by describing a reference signal that has this modulation depth. To describe this signal, we first need an auxiliary signal $\mu_{b}$ that is fully modulated by a cosine term. It is given by the equation below.

$$
\mu_{b}\left(n, f_{m, b}\right)=\sqrt{\frac{2}{3}} \cdot\left[1+\cos \left(2 \pi \frac{f_{m, b}}{f_{s}} n+\varphi_{b}\right)\right] \cdot \lambda_{b}(n) .
$$

Here, $n$ is the sample index, $f_{s}$ is the sampling rate, $f_{m}$, $\mathrm{b}$ is the modulation frequency in subband $b$, and $\lambda_{b}$ is a band-limited stationary signal with a constant envelope over time (which can in some cases only be approximated, but is indeed achieved with the binary signals we will discuss later). Considerations on the signal's band limits will follow further below.

Using the auxiliary signal $\mu_{b}$, the reference signal, $\sigma_{b}$, can be constructed, as described by the equation below.

$$
\sigma_{b}\left(n, f_{m, b}\right)=a_{b} \cdot \mu_{b}\left(n, f_{m, b}\right)+\left(1-a_{b}\right) \cdot v_{b}(n) .
$$

Here, the signal $v_{b}$ is again a band-limited stationary signal with constant envelope and shall have the same RMS as the signal $\lambda_{b}$, and the factor $a_{b}$ is the link to the modulation depth via the following equation:

$$
a_{b}= \begin{cases}1-10^{-\frac{1}{20}\left(m_{b} / \mathrm{dB}\right)} & ; m_{b}<M_{\max } \\ 1 & ; m_{b}=M_{\max }\end{cases}
$$

\subsection{Definitions related to verification}

- Test coverage would ideally describe the percentage of the system's input or state parameter range used during tests. In the case of a signal processing system dealing with quasi-continuous signals, the range of possible input signals is dramatically large and has to be constrained to a moderate number of test signals for practical testing. The selection of tests is here based on the hypothesis that one test signal from a certain class - an equivalence class - is sufficient to test the whole class. This hypothesis shall be called uniformity hypothesis [18] in the following. Test coverage in the context of this article denotes the percentage of equivalence classes rather than the percentage of possible signals that is reached by the test. State space coverage is not considered.

- A test step is a time interval during a test (definition based on [8]).

- A test sequence is a composition of test steps that cover certain equivalence classes, optionally together with a specification of transitions between them. Note that for simplicity, this article does not distinguish between test sequences and test cases, as does [8].

\section{Verification procedure}

The procedure is applicable to signal processing systems that can be characterized by observing their output in dependency of systematically chosen input signals. The procedure has three steps to be described in the following sections:

1) Identifying the requirements against which to test

2) Designing tests

3) Performing tests

\subsection{Identifying requirements against which to test}

Requirements engineering (e.g., [19]) typically ensures that testable requirements are available. However, this matter will not be covered here in more detail, because it is not actually relevant for this article how the requirements specification has been established. Here, it is important to have such a specification and, based on it, identify those requirements that are within the scope of the test.

\subsection{Designing tests}

3.2.1 Describing abstract test sequences with regard to test coverage

Test design should use a method that can ensure the desired test coverage. In the domain of signal processing systems, we propose the classification tree method for embedded systems (CT/ES) from $[7,8]$ : the input domain of the system under test is partitioned into equivalence classes according to the original classification tree method from [20], then test sequences are defined in order to cover them with test steps that are abstract, i.e., independent of concrete test signals.

Finding suitable equivalence classes is a key to an appropriate test design; therefore a good starting point is helpful. We expect the identified requirements according to Sect. 3.1 to be a suitable starting point, because they may give hints about the most important input parameters that have to be considered in 
partitioning the system's input domain. A main reason for this: we expect the main functionality of the signal processing systems targeted here to be a processing of input signals. The requirements thus have to specify how these input signals have to be processed and thus make statements about the system's input domain.

The classification tree representation of equivalence classes, test sequences and test steps enables the assessment of test coverage and the further elaboration on the test, i.e., the verification of the test design and the synthesis of concrete stimuli that comply to it by covering the corresponding equivalence classes of the system's input space.

It may not be possible to have the classification tree method cover all system parameters specified by the requirements according to Sect. 3.1. Therefore the test designer should also identify those tests that are needed in addition to the ones from the identified test sequences in order to verify each requirement with at least one test.

\subsubsection{Selecting the synthesis procedure for implementing the concrete test signals}

This section discusses different stimuli from system identification and their use as a basis for synthesizing concrete test signals that match the abstract signal description as to the previous section. These signals should be designed for real systems whose usable signal range has its lower limit in a noise floor and its upper limit at a certain maximum level that is given by limited word lengths in the digital domain and/or limited amplitudes in the analog domain. Ideal stimuli would therefore have a white power spectrum, such that the spectral components of the background noise are negligible compared to those of the stimulus at any frequency. To provide good signal-to-noise performance within the given level limitations, the peak factor [21] of the stimulus should also be small. Obviously, binary signals have a minimum peak factor, but are reported to oppose challenges to some digital-to-analog converters [22] and cannot match every given power spectrum. Therefore, different kinds of signals will be considered in the following.

- Discrete-interval binary signals [23,24] result from algorithms that search a certain set of continuoustime binary signals for those ones whose power spectrum approximately matches a specified one. We define that a discrete-interval binary sequence (DIBS) is the discrete-time representation of a discrete-interval binary signal.

- Binary maximum length sequences (binary msequences) contain all possible sequences of storage initialization in a binary shift register of length $L$, except the initialization of all storages with zero - resulting in a sequence length of $2^{L}-1$ [22]. For system identification they are usually synthesized with computer programs $[25,26]$ rather than with shift registers. Binary $\mathrm{m}$-sequences are perfect sequences and have a minimum peak factor.

- A periodic multi-sine signal with a predefined discrete power spectrum can be obtained by adding sine waves of different frequencies. Their amplitudes result from the desired spectrum; phases, however, can be varied, e.g., for minimizing the signal's peak factor $[21,23,27,28]$. Signal synthesis is most efficiently done using Fast Fourier Transform methods $[29,30]$.

While DIBS are based on iterative approximation of the desired power spectrum, the synthesis of multi-sine signals usually presets the amplitude spectrum and bases its optimizations on varying the phase. As a consequence, the synthesis of multi-sine signals usually reaches the desired power spectrum quite precisely, whereas DIBS can lack precision, particularly regarding the synthesis of band-limited signals. Figure 3 illustrates this in showing the spectrum level of a DIBS that has been synthesized according to the prescription of a band-limited white power spectrum in the band limits of a typical noise reduction subband around $1 \mathrm{kHz}$. The signal indeed has an approximately white power spectrum in this subband, but around $4 \mathrm{kHz}$ there is a high amount of side-lobe energy, as indicated by the arrow in Figure 3.

Sect. 4.5 exemplarily demonstrates the different stimuli that have been described with sample measurements. Their performance in these measurements will be discussed in Sect. 4.6. A more general discussion of stimuli can be found in the literature of system identification (e.g., [29]).

\subsubsection{Selecting the measurement technique}

There are different techniques for measuring the frequency response $H(f)$ of the system under test: for example, the measurement based on the adaptive LMS algorithm (Figure 2 bottom left) of $H(f)$ and the straightforward computation of an estimate of $H(f)$ from the signals $x$ and $y$ based on the DFT (Figure 2 bottom right).

The impulse response of a system under test can be time varying and it may be desired to track the corresponding variations over time. The NLMS algorithm can achieve this under certain conditions and is therefore a common choice in transfer function measurements (e.g., [13]).

The DFT-based measurements require a steady-state condition of the system under test. The used test signals should have spectral components that are constant [16] over the frequency range of interest. They should also be periodic [4], which can avoid leakage errors [31] in processing based on the DFT, if the DFT window length 


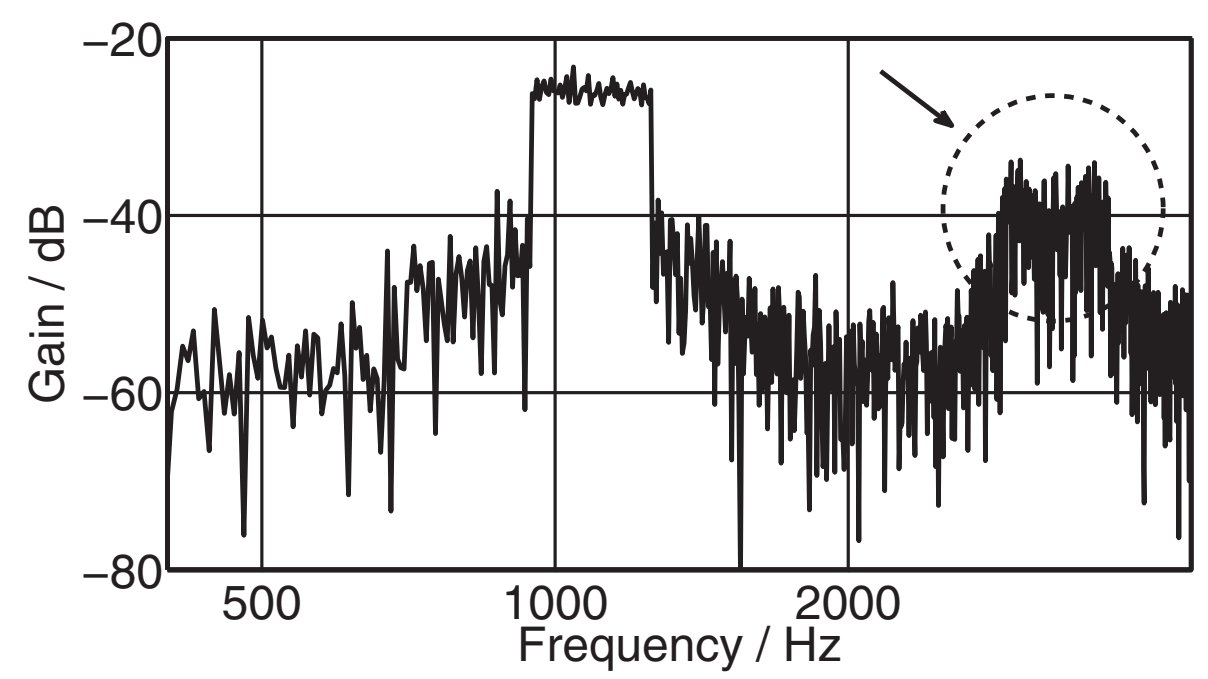

Figure 3 Spectrum level of a sample DIBS.

is a multiple of the period length [29]. If this match of lengths is not possible, zero stuffing - the insertion of additional zeros into the DFT frame - can adjust the signal frame to the DFT frame. It has been shown, however, that this may reduce the measurement precision compared to a situation with matched lengths [32]. As a consequence, it shall be a prerequisite for all further considerations about DFT-based processing that the DFT window length matches the period length of the used stimulus. In case of signals whose length is not a power of two, this may mean that the Fast Fourier Transform algorithm cannot be used. Even in these cases, we expect the computation time to be sufficiently short, based on the assumption that measurement data will be post-processed with the computational power of a modern desktop computer.

Both LMS-based and DFT-based measurements ideally need stimuli with a white power spectrum. The DFTbased measurements only have optimum precision when used with periodic stimuli, whereas the LMS does not require periodicity of signals. The most important criterion for selecting the measurement approach is the time variance of the system under test: while LMS-based measurements can handle time variance under certain conditions, the DFT-based measurements only work with a time-invariant system. DFT-based measurements have the advantage that no convergence of an iterative algorithm is needed. This makes the measurement window for a given frequency resolution small and thus the time resolution high.

\subsection{Performing tests}

How to perform the tests is dependent on the chosen test design. We can therefore not state a general flow of activities for this part of the procedure. We rather use typical experiments to demonstrate the step of performing tests. This will be done within the next section.

\section{Measurements}

This section demonstrates the application of the verification procedure in the hearing instrument domain, based on experiments with hearing instruments. The design of experiments is given by the proposed verification procedure. The device under test and the measurement setup will be presented in the following sections.

\subsection{Device under test}

In all experiments, the device under test was a hearing instrument with a modulation-based noise reduction subsystem. Most of the devices used for the experiments below were part of recent test plans at the Bernafon laboratories, allowing us to perform most of the shown experiments within the regular test plans of the laboratory. As a consequence, different experiments have been performed with different hearing instrument models, because test plans do not necessarily foresee to sequentially perform all test cases with the same one. The noise reduction subsystems of the used hearing instruments were equivalent and thus satisfy the same requirements and design.

The requirements toward the noise reduction subsystem under test have been stated in Table 1, using "shall" clauses, which are a common practice in requirements engineering [19]. Table 1 identifies each of them with a unique code (ID) to be used for further reference in this article, but also to show the hierarchy of requirements (e.g., requirement 1.1.1 adds more detail to requirement 1.1). Literature references in the rightmost column of 


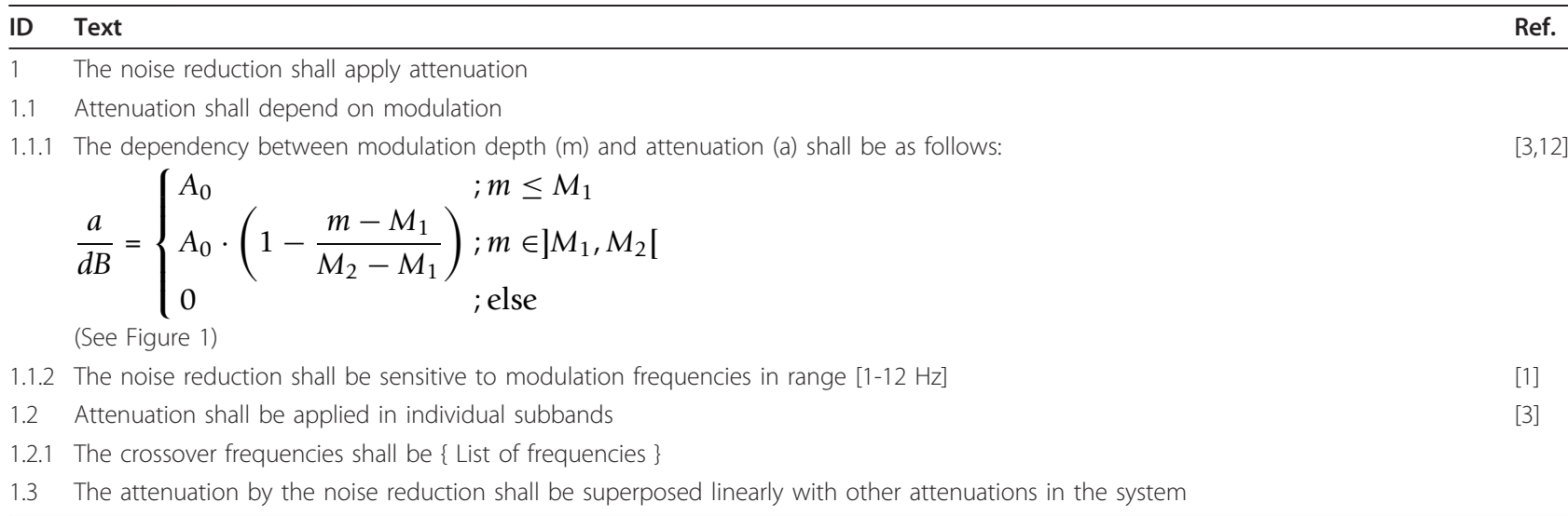

Table 1 indicate sources of the information contained in the corresponding requirement.

The design of the investigated noise reduction subsystem is shown in Figure 4:

- The gray blocks compose a functional model of the noise reduction subsystem in the notation of the Simulink ${ }^{\circledR}$ software.

- The unfilled blocks indicate the IDs of requirements from Table 1 that are fulfilled by the associated functional blocks.

The functionality of the noise reduction subsystem according to Figure 4 is explained in [3] and will only be briefly summarized here: The block "Filter" extracts subband contents of the input signal for each subband individually and feeds these into block "Compute modulation" to estimate modulation depths according to Equation 2. The block "Compute attenuation" determines attenuation as a function of modulation depth according to Figure 1. This attenuation will be applied in block "Apply attenuation" together with other attenuation in the system, which was zero for all experiments except the last one where it resulted from a transient noise reduction system to be described later. The block "Synchronize" ensures that the signal in the lower signal path is delayed by the group delay of the upper path in order to ensure that the processing in block "Apply attenuation" will be based on correctly timed information.

\subsection{Test setup}

The setup for performing the designed test consisted of a combination of off-the-shelf hardware and software as well as customized computer programs. This section describes each of them.

\subsubsection{Infrastructure for test design}

An abstract description of test sequences was done using the tool $\mathrm{CTE}^{\circledR}[33,34]$ that supports the earliermentioned CT/ES method. The MATLAB ${ }^{\circledR}$ technical

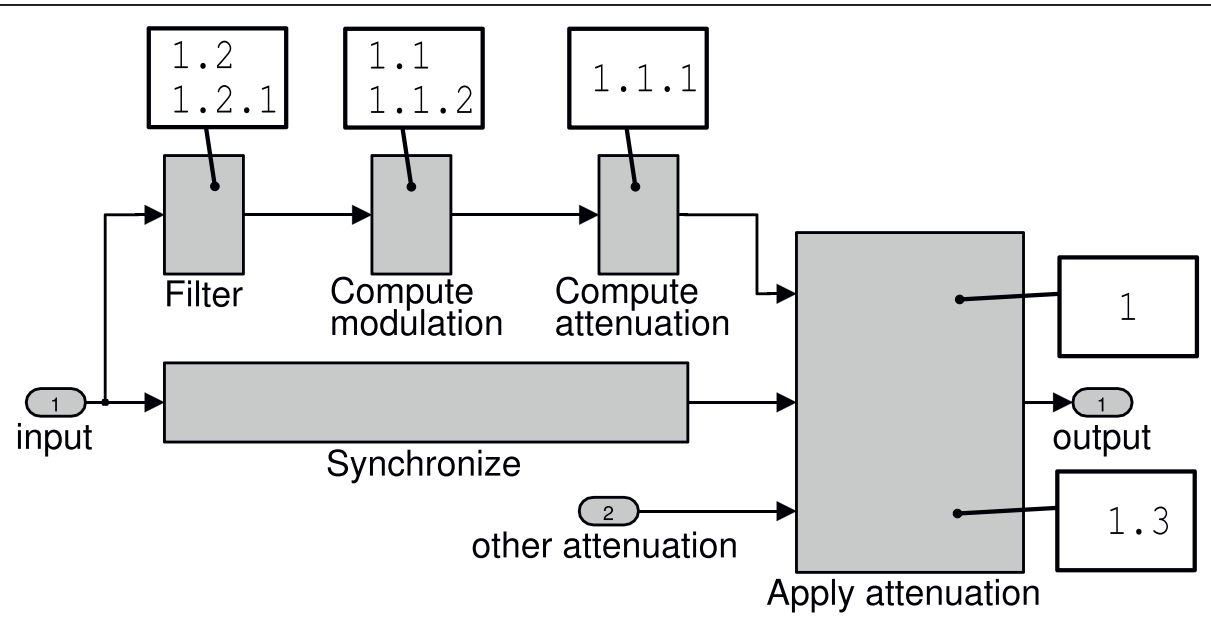

Figure 4 Functional model of a noise reduction subsystem according to [3], annotated with the requirements from Table 1 to be fulfilled by the different blocks. 
computing environment was used to synthesize binary m-sequences according to [26]. One of its third-party toolboxes, the Frequency Domain Identification Toolbox (FDIDENT, [35]), was used for synthesizing discreteinterval binary sequences based on [23] and multi-sine signals according to [28].

\subsubsection{Infrastructure for test execution}

For all shown results, the test setup was the same: A test system was prepared for making measurements with synthetic test signals. Figure 5 illustrates the setup: The hearing instrument under test was located in an off-the-shelf acoustic measurement box with a loudspeaker $\left(L_{1}\right)$ for presenting test stimuli to be picked up by the hearing instrument's input transducer $\left(M_{2}\right)$. The hearing instrument's output transducer $\left(L_{2}\right)$ was coupled with a measurement microphone $\left(M_{1}\right)$ so tightly that environment sounds can be neglected in comparison to the hearing instrument's output. The coupler is a cavity that mimics the human ear canal. Here, we used a socalled 2cc-coupler.

Note that the described test setup differs from the usual condition in which a hearing instrument is worn, because the effect of the human head on the sound field from the sound source is not taken into account. The acoustic effect of the human head in wearing the hearing instrument has thus been neglected here, but it could easily be modeled by putting the hearing instrument under test on an artificial head within the test box.
The test system [36] was implemented using a National Instruments PXI ${ }^{\mathrm{TM}}$ system running customized computer programs based on National Instruments LabVIEW. The test system was equipped with a NI-PXI 4461 analog input/output card that can play test signals originating from a hard disk, where they have been stored after creating them with the MATLAB ${ }^{\circledR}$ technical computing environment. The signals were presented via a digital-to-analog converter (D/A) of the input/output card, an audio amplifier (Amp. A) and the loudspeaker of the measurement box $\left(L_{1}\right)$, while recording the hearing instrument's output via the measurement microphone $\left(M_{1}\right)$, a microphone pre-amplifier (Amp. B) and an analog-to-digital converter (A/D) of the input/output card.

The recorded digital data were stored in a file on a hard disk that could be read by the MATLAB ${ }^{\circledR}$ technical computing environment for further processing. The sampling rate for both playing and recording signals was set to $22,050 \mathrm{~Hz}$. The test system ensured synchronous playback and recording.

\subsection{Test design}

Testing a modulation-based noise reduction system should observe attenuation in different subbands as a function of modulation. Figure 6 uses the CT/ES method's proposed graphical notation of classification trees to show the partitioning of hearing instrument input

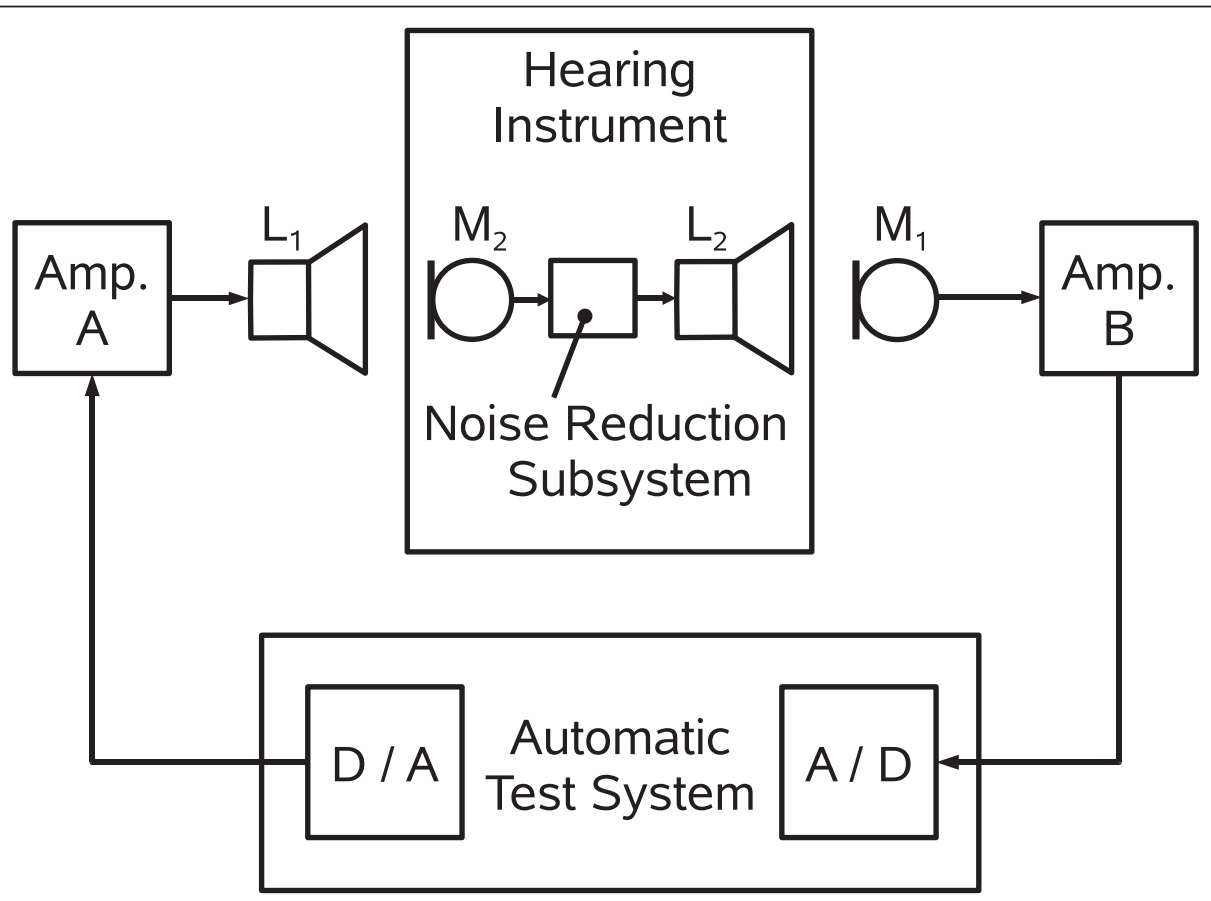

Figure 5 Measurement setup. 


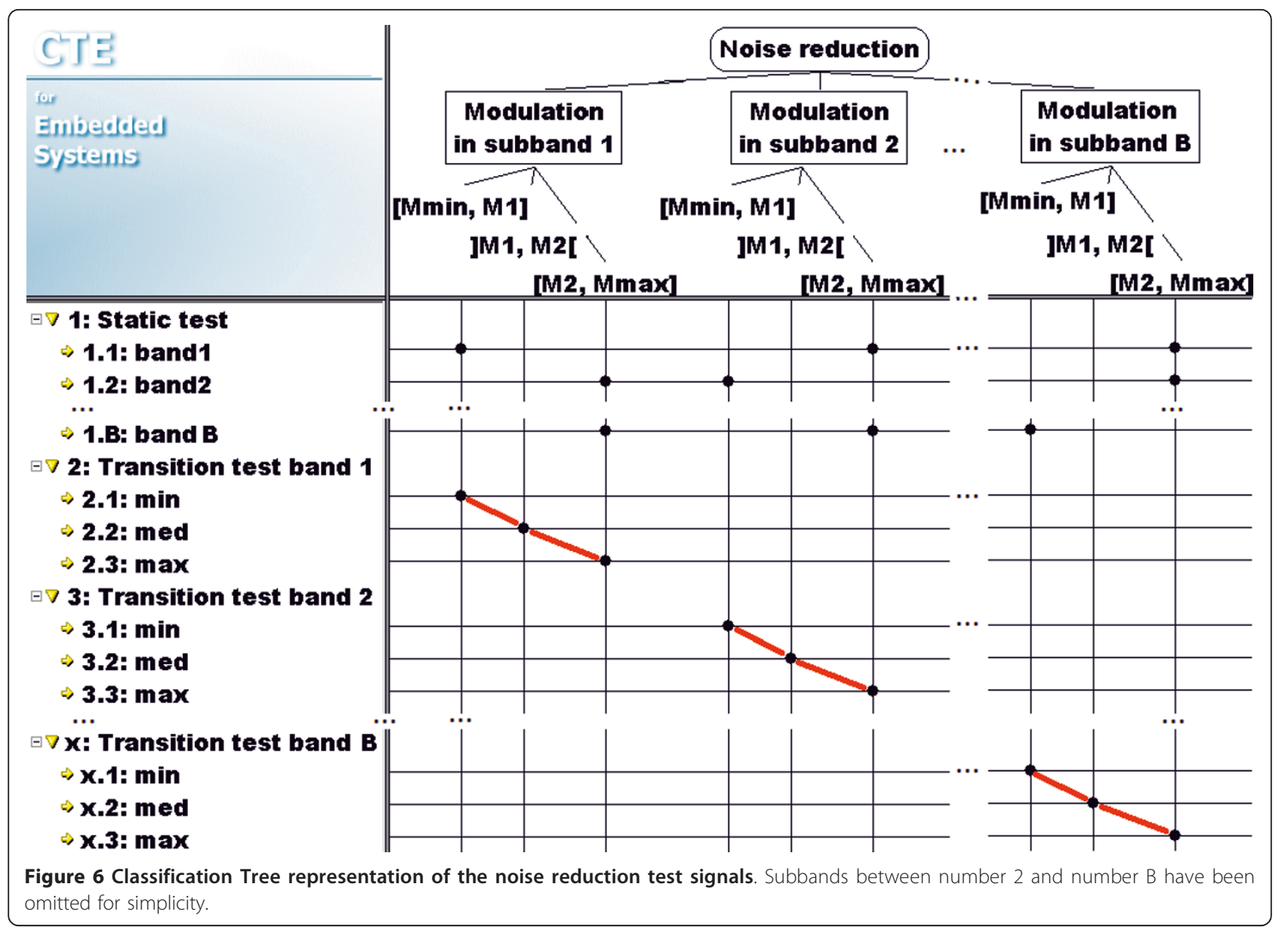

signals into equivalence classes as a basis for testing a multi-band noise reduction system with modulationdependency:

- Input parameters (symbolized by rectangles) are the modulation depths (brief: modulations) in the different subbands, based on requirement 1.1 and 1.2 in Table 1.

- Equivalence classes (symbolized by range expressions in square brackets) have been derived from requirement 1.1.1 in Table 1.

- Test sequences ("1", “2”, “3”,...) and test steps ("1.1", "1.2",...) are denoted by short verbal descriptions in the column on the left.

- Filled circles on the grid show that a test step should cover a certain equivalence class.

- A diagonal straight line between two circles denotes a gradual transition of the used test signal between different equivalence classes.

The circles and their connection lines in Figure 6 are an abstract description of test signals that should be suited for verifying most multi-band modulation-based noise reduction systems.

Figure 6 shows two kinds of test sequences: On the one hand, a static test (1) that covers the extreme modulation classes of very low and very high modulation for all subbands, and on the other hand dynamic tests ( 2 to $x$, one per subband) that gradually vary modulation within the intermediate modulation range $] M_{1}, M_{2}$ [. Together, these test sequences achieve sufficient test coverage: since all equivalence classes have at least one circle vertically below them, all equivalence classes are covered by tests.

The abstract test description from Figure 6 should now be mapped to concrete test signals that are used for frequency response measurements based on a suitable measurement technique. Although NLMS-based measurements are a common way of measuring acoustic frequency responses (e.g., [13]), we chose DFT-based measurements, because of the possibility to achieve high time resolution, which were required in one of the experiments. As a consequence, test signals had to be periodic.

When used as a stimulus for subband measurements, a periodic test signal needs to have its power 
concentrated in the frequency range of interest, and the most simple assumption is that it should approximate band-limited white noise. Some synthesis algorithms require the absolute values of Fourier coefficients of the signal as an input. If the desired period length in samples is $N$, and frequency range of interest is from $f_{1}$ to $f_{2}$ (where $f_{1}>0$ and $f_{2} \geq f_{1}+N^{-1} \cdot f_{s}$ ) and the desired RMS of the synthesized signal is $r$, then the target values for the synthesis algorithm are given by the following absolute values of Fourier coefficients $\frac{c}{k}$ (based on [4]):

$$
\left|c_{k}\right|=\left\{\begin{array}{l}
\frac{r}{\sqrt{2\left(\left\lceil N \cdot \frac{f_{2}}{f_{s}}\right]-\left\lfloor N \cdot \frac{f_{1}}{f_{s}}\right\rfloor+1\right)}} ;\left|\frac{N \cdot f_{1}}{f_{s}}\right| \leq|k| \leq\left\lceil\frac{N \cdot f_{2}}{f_{s}}\right\rceil \\
0 \quad \text { else }
\end{array}\right.
$$

Since system tests will acoustically stimulate the system under test, we would theoretically have to describe acoustic signals here, which are in continuous time. However, since the native format of the given test system is a digital waveform, we describe signals in discrete time. All stated sampling rates refer to the test system, not to the system under test.

Let $B$ be the number of subbands, and let the lower and upper crossover frequency of subband number $b$ be $f_{c, b}$ and $f_{c, b+1}$ respectively. Furthermore, let the signal $\sigma_{b}$ be the reference signal from Equation 4. The signals in that equation shall be constructed as follows: the signal $v_{b}$ shall have a Fourier spectrum that approximates the one from Equation 6 with $f_{1}=f_{c, b}$ and $f_{2}=f_{c, b+1}$. The signal $\mu_{b}$ be the one from Equation 3 where the signal $\lambda_{b}$ is synthesized the same way as $v_{b}$, but with $f_{1}=f_{c, b}+$ $f_{m, b}$ and $f_{2}=f_{c, b+1}-f_{m, b}$ [4]. Then, the following equation defines a test signal $\theta_{b}$ that has configurable modulation in subband number $b$ and maximum modulation in the other subbands:

$$
\theta_{b}(n)=\sigma_{b}\left(n, f_{m, b}\right)+\sum_{i \in(\{1,2, \ldots B\} \backslash\{b\})} \mu_{i}\left(n, f_{m, i}\right) .
$$

The parameter $\phi_{b}$ on which the above signal depends via Equation 3 was left variable to allow for experimenting with different values of it.

The test steps from Figure 6 never require more than one subband at a time to have a modulation outside the range $\left[M_{2}, M_{\max }\right]$. Using only maximum modulation to cover the equivalence class of that range, one can use the signal $\theta_{b}$ from Equation 7 to establish all test steps from Figure 6, if a suitable modulation of signal $\sigma_{b}$ is chosen in the one subband whose modulation falls into another equivalence class (note that for each test step in Figure 6, there is maximum one definition of such a subband).

So far, the described stimuli therefore cover all requirements from Table 1, except number 1.1.2, 1.2.1 and 1.3. These requirements can be covered with a simple measurement approach that does not require an abstract test design. This will be demonstrated in Sect. 4.5 .

\subsection{Test procedure}

For all experiments, the gain in the hearing instrument under test was set $20 \mathrm{~dB}$ below the maximum offered value to reduce non-linearities. Unless stated differently, all adaptive features of the hearing instrument, apart from noise reduction, were turned off for all test runs. The hearing instrument was furthermore configured for linear amplification, this means that there was no dynamic range compression.

Before each experiment, the test system was calibrated using built-in functionality, in order to ensure that transfer characteristics of all equipment in the signal path, particularly the acoustic transducers, were compensated in the digital signal processing of the test system. This ensured that the power spectra encoded in audio files of the input and output signals were equivalent to the acoustic power spectra at the input and output transducers of the device under test.

According to the earlier-mentioned differential measurement approach, two DFT-based measurements were performed per stimulus: first with the noise reduction subsystem of the hearing instrument switched off, and second while having it switched on.

Only the output-related DFT spectra $Y_{k}^{(\text {on })}(n)$ of the system with noise reduction enabled and $Y_{k}^{(\text {off })}(n)$ of the system with noise reduction disabled were recorded to compute the noise reduction $\mathrm{s}$ transfer function $H_{k}^{\text {(subsystem) }}$ by the following equation that results from the definition of the differential measurement in Sect. 2.1.2, from Equation 1 and from the fact that the input signal was the same for both measurements:

$$
H_{k}^{\text {(subsystem) }}(n)=\frac{y_{k}^{(\text {on })}(n)}{Y_{k}^{(\text {off }}(n)}
$$

In using the above equation, measurement samples $Y_{k}^{(\text {off })}(n)=0$ would have been treated as invalid samples and discarded from the result to avoid division by zero, though in practice, such samples did not occur during the experiments that were made.

\subsection{Experiments}

\subsubsection{Verification of crossover frequencies}

The objective of the test described in this section was the verification of requirement 1.2.1 from Table 1, thus to verify the crossover frequencies between the noise 
reduction subsystem's subbands. We separately defined a signal used for driving the noise reduction subsystem into a desired state and one used for measuring the system's frequency response, which - in combination yielded the test signal according to the equation below.

$$
s(n)=\left\{\begin{array}{ll}
a \cdot \sin \left(2 \pi \frac{f_{0}}{f_{s}} n\right)+b \cdot\left[1+\cos \left(2 \pi \frac{f_{m}}{f_{s}} n\right)\right] \cdot p(n) & ; \frac{n}{f_{s}} \leq T \\
p(n) & ; \text { else }
\end{array} .\right.
$$

The signal $p$ was chosen to be a periodically repeated binary $\mathrm{m}$-sequence of 1,023 samples period length, and the time $T$ that passes between the start of the measurement and the application of the signal $p$ was set to $40 \mathrm{~s}$. The inherent assumption of this procedure is: after time $T$ the noise reduction subsystem has settled to steady state and maintains it while the signal s continues, such that a frequency response can be measured, based on the stimulus $p$. The signal applied before time $T$ contains a pure tone to stimulate the noise reduction subband around frequency $f_{0}$, added to a modulated signal that is constructed in a way similar to Equation 3. Based on empirical investigation of the procedure, the parameters $\mathrm{a}$ and $\mathrm{b}$ were chosen such that the pure tone's level was $15 \mathrm{~dB}$ higher than the level of the remaining signal components before time $T$, in order to make the unmodulated signal the dominant stimulus around frequency $f_{0}$. The modulation frequency was set to $f_{m}=$ $4 \mathrm{~Hz}$, because this is the frequency at which the modulation spectrum of speech has its peak [1].

The frequency $f_{0}$ was varied stepwise within the bandwidth of the system under test. The step width was chosen between 40 and $250 \mathrm{~Hz}$, depending on the bandwidth of the noise reduction subbands in the given frequency region.

For each measurement, the signal $p(n)$ was the input signal of the system under test between time $T$ and the end of the measurement, allowing for frequency response measurements. Differential DFT-based measurements were performed to obtain the frequency response of the noise reduction subsystem immediately after time $T$. Averaging of the data resulting from three subsequent periods of the measurement stimulus was used for obtaining a smoothed frequency response [37]. The measured frequency responses were postprocessed by a human observer: it was necessary to discard duplicate responses of the same subband as well as invalid measurements that were caused by $f_{0}$ inbetween two sub-bands triggering the noise reduction subsystem in both of them. Afterward, the observer could determine crossover frequencies by graphically intersecting the frequency responses of two adjacent subbands.

Figure 7 shows some examples of the responses that were measured. Subfigures a and b show cases in which more than one noise reduction subband was triggered by the pure tone of frequency $f_{0}$. The frequency responses of such cases all had the shown characteristic shape for the given noise reduction subsystem, allowing the observer to identify such measurements and ignore them. Figure 7c, $d$ are examples of valid measurements: the intersection of their graphs would show that the system under test has a crossover frequency at approximately $1,550 \mathrm{~Hz}$. To result in a passed test, the set of all obtained frequencies was verified to match specified corner frequencies of the noise reduction sub-bands within a specified tolerance. Figure 8 shows how the absolute value of the difference between the specified and the measured crossover frequency was distributed over eleven valid measurements from the given experiment with an error-free system under test. The distribution shows that a typical $10 \%$ tolerance band would have resulted in a passed test, but even a $5 \%$ tolerance band would have been possible in the given case.

\subsubsection{Verification of the frequency response for a static modulation pattern}

This section describes the "static test" according to Figure 6, that means a measurement performed with a signal composed of strongly modulated signals with a modulation depth in range $\left[M_{2} ; M_{\max }\right]$ in all subbands except one - subband number $b$, in which the modulation is in the range $\left[M_{\min } ; M_{1}\right]$. Based on Sect. 4.3, the signal $\theta_{b}$ from Equation 7 was used as the test signal. The system of equations formed by Equations 7, 3 and 4 was used as the basis for signal synthesis, and the parameters of these equations were set to $a_{b}=0$ (thus choosing $M_{\min }=0$ as the modulation depth for subband number $b$ ) and $f_{m}=5.4 \mathrm{~Hz}$ [4]. The remaining parameter $\phi_{b}$ was varied between the different subbands and between the different measurements between different empirically found values. The signals $\lambda_{b}$ and $v_{b}$ in the resulting equation were synthesized with a level of 70 dB SPL and a period length of 4,096 samples either as multi-sine signals or as signals of type DIBS. Table 2 lists the measurements that were performed and the corresponding choice of parameter $\phi_{b}$ and the signal type of the mentioned signals.

For each measurement, the test stimulus was presented during at least $15 \mathrm{~s}$. Differential measurements of the noise reduction subsystem's frequency response were made, averaging five DFT windows. These windows were taken from the last $5 \mathrm{~s}$ of the test run in order to observe the steady-state condition.

Figure 9 shows the measurement results together with a reference response (Figure 9a), which has been obtained by using a functional model of the block "Apply attenuation" from Figure 4 in the condition in which it applies zero attenuation to all subbands but $b$, and maximally attenuates that subband. The $3 \mathrm{~dB}$ 

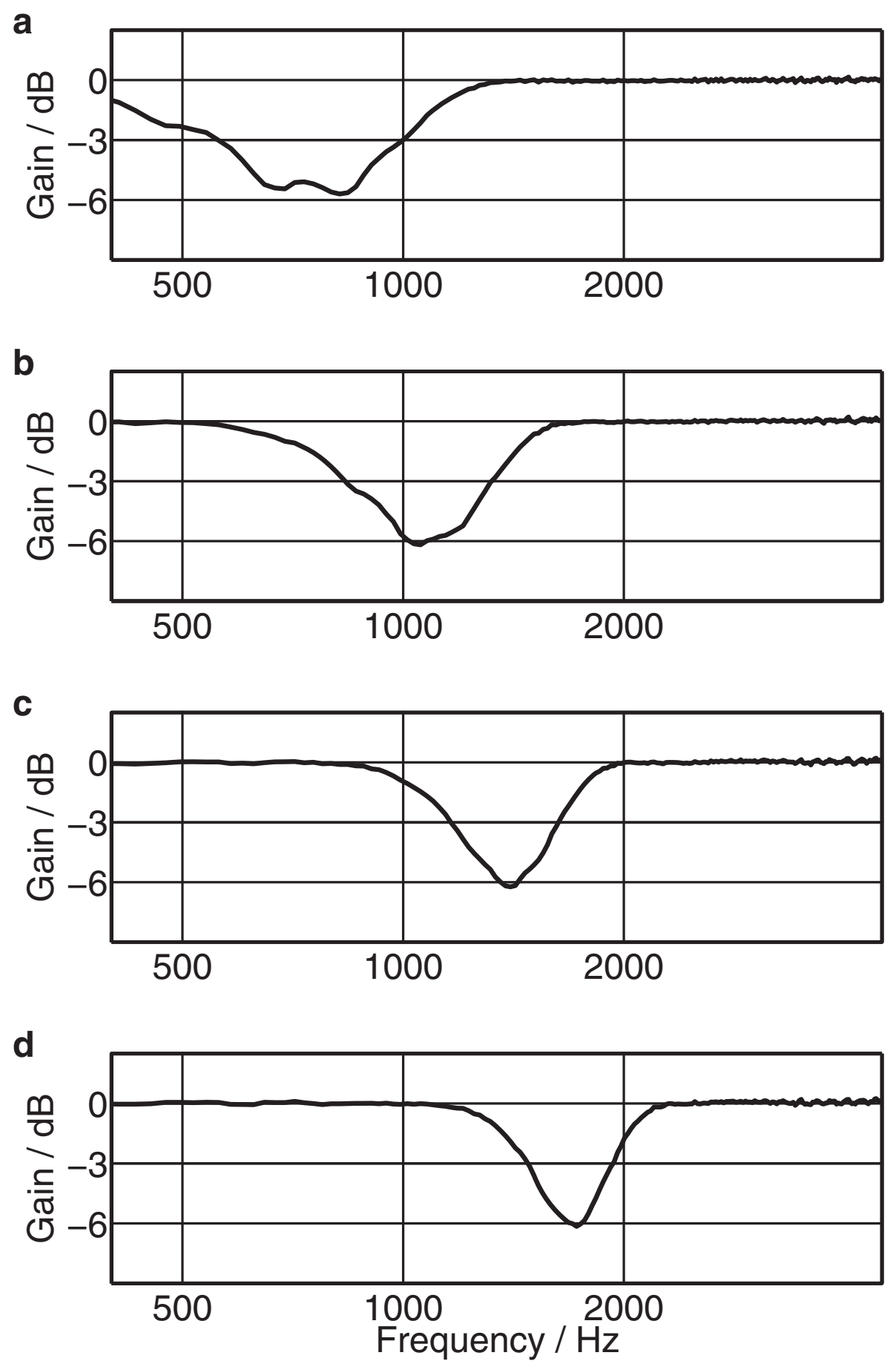

Figure 7 Determining crossover frequencies of the noise reduction subsystem: (a) $f_{0}=750 \mathrm{~Hz}$, (b) $f_{0}=1,000 \mathrm{~Hz}$, (c) $f_{0}=1,480 \mathrm{~Hz}$, (d) $f_{0}=$ $1,600 \mathrm{~Hz}$

corner frequencies of the simulated subband $b$ have been indicated with dashed lines.

Observations: From the measured responses, only the one of Figure $9 \mathrm{~d}$ has the correct subband attenuation in the sense that it produces the expected $3 \mathrm{~dB}$ corner frequencies. Furthermore, Figure $9 \mathrm{~b}$ shows a side effect, the attenuation in a subband not adjacent to subband number $b$ (as indicated with an arrow on the figure). 


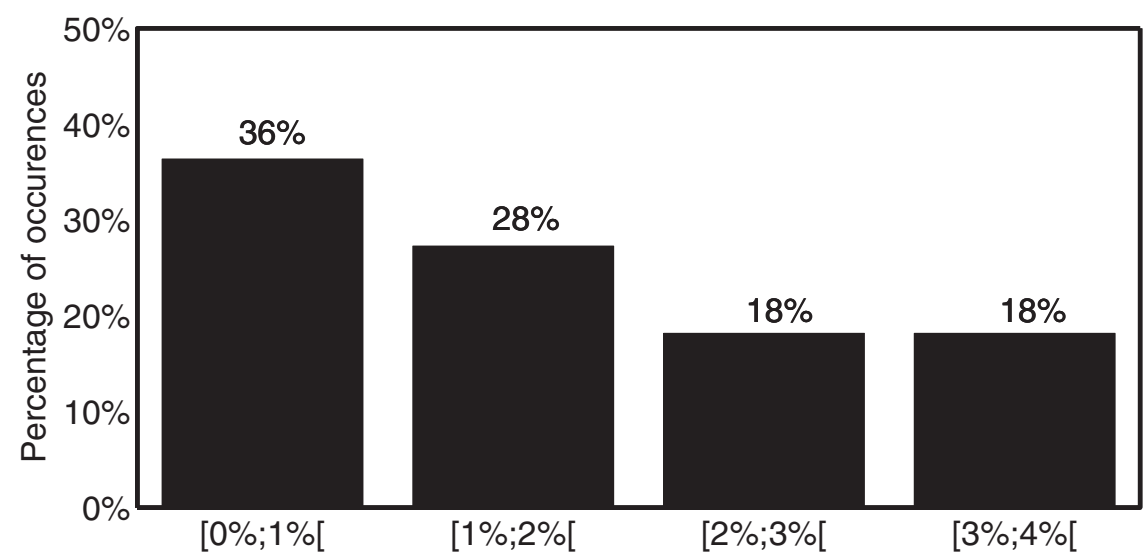

Relative deviation of measured frequency from specified frequency

Figure 8 Measured versus specified crossover frequencies.

\subsubsection{Investigation of the side effect in the DIBS-based measurement}

Measurement data from different runs of measurement number 1 according to Table 2 with different hearing instruments were analyzed in order to investigate the side effect according to Figure 9b. The hearing instruments had been set to different noise reduction configurations during the measurements. The measurement data were subdivided according to the sensitivity to unmodulated noise of the used noise reduction configuration. The higher the configured first knee point " $M_{1}$ " according to Figure 1, the more sensitivity to unmodulated noise was assumed in the classification. As a result, three classes were obtained: a "Low" class for low sensitivity, a "Med" class for medium sensitivity, and a "High" class for high sensitivity.

The data from different runs of the "static test" according to Figure 6 were plotted in graphs like the ones of Figure 9, and these were inspected visually. For each graph, the observer counted frequency bands not adjacent to subband $b$ whose peak attenuation was more than $1 \mathrm{~dB}$, thus bands with the side effect according to Figure $9 \mathrm{~b}$. The counted value was divided by the total number of subbands $B$, and the result was multiplied by 100 , resulting in a percentage of subbands with the side effect per hearing instrument per class of sensitivity to unmodulated noise. The results of this analysis are given in Table 3 for five sample hearing instruments that were known to have correctly operating noise reduction subsystems from other verification activities not in the scope of this article. It can be observed that the percentage of side effects increases from the "Low" class to the "High" class for every observed hearing instrument.

4.5.4 Verification of attenuation with varying modulation

Attenuation function measurements are needed to cover the "transition tests" according to Figure 6. They should characterize a modulation-based noise reduction system by measuring its typical performance characteristic according to Figure 1. Modulation depth can be varied by changing parameter $a_{b}$ in Equation 4 . This was done by varying parameter $m_{b}$ in Equation 5 from $0 \mathrm{~dB}$ to 20 $\mathrm{dB}$ in steps of $2 \mathrm{~dB}$. A test signal $\tilde{\theta}_{b}$ was constructed as a modified version of signal $\theta_{b}$ from Equation 7 and was used for the measurements. One modification (according to [4]) was to choose a sum index range of $(\{1,2, \ldots$ $B\} \backslash\{b-1, b, b+1\})$ instead of $(\{1,2, \ldots B\} \backslash\{b\})$. The subbands that had been removed from the sum index range were instead targeted with signals of variable modulation according to Equation 4. The modification should ensure that the stimulus of variable modulation spanned

Table 2 Parametrization of measurements

\begin{tabular}{cll}
\hline Measurement number & Choice of parameter $\phi_{\mathbf{i}}$ as a function of subband number $\boldsymbol{b}$ & Type of signal $\lambda_{\boldsymbol{b}}$ and $\boldsymbol{v}_{\boldsymbol{b}}$ \\
\hline 1 & $\forall_{i: \phi_{i}}=0$ & DIBS \\
2 & $\forall_{i} \phi_{i}=0$ & multi-sine \\
3 & $\varphi_{i}=\left\{\begin{array}{l}\frac{\pi}{2} ; i \neq b \\
0 ; i=b\end{array}\right.$ & multi-sine \\
\end{tabular}




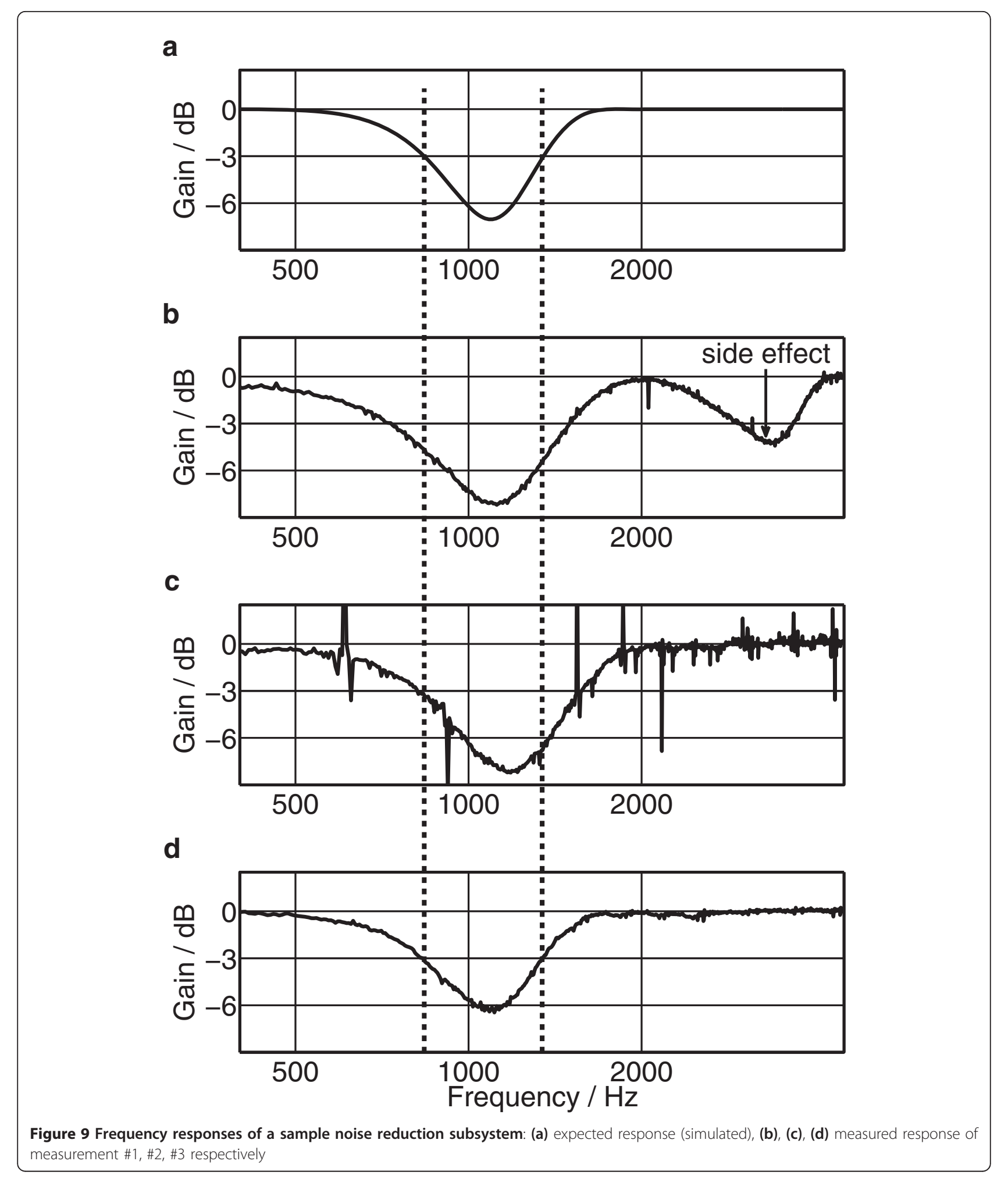

more than one subband of the noise reduction subsystem under test, thus making the procedure invariant to non-ideal subband split. The mentioned changes are consistent with the test design (Figure 6), whose "transition test" sequences do not constrain the modulation of any subband except the one with the specified variation of modulation depth. Another modification of signal $\theta_{b}$ in constructing signal $\tilde{\theta}_{b}$ was to filter out side 
Table 3 Side effects in dependency of modulation sensitivity

\begin{tabular}{cccc}
\hline Hearing instrument & \multicolumn{2}{c}{ Percentage of subbands with side effect } & \\
\cline { 2 - 4 } & in "Low" class (\%) & in "Med" class (\%) & in "High" class (\%) \\
\hline 1 & 0 & 0 & 47 \\
2 & 0 & 7 & 60 \\
3 & 0 & 0 & 60 \\
4 & 0 & 7 & 67 \\
5
\end{tabular}

lobe influences from the subband of interest [4]: if subband number $b$ is this subband, then one can eliminate the influences from other subbands by using a bandstop filter whose band limits are outside of subband number $b$ (here, we chose each of them in the middle of one subband adjacent to $b$ ). Let $h$ be the impulse response of such a band-stop filter and let "*” be the convolution operator. Then, $\tilde{\theta}_{b}$ can be constructed according to the equation below.

$$
\tilde{\theta}_{b}(n)=\tilde{\sigma}_{b}(n)+h(n) * \sum_{i \in(\{1,2, \ldots B\} \backslash\{b-1, b, b+1\})} \mu_{i}\left(n, f_{m, i}\right) .
$$

Here an auxiliary signal $\tilde{\sigma}_{b}$ was used according to the following definition:

$$
\tilde{\sigma}_{b}(n)= \begin{cases}\sigma_{b}(n)+\sigma_{b+1}(n) & ; b=1 \\ \sigma_{b-1}(n)+\sigma_{b}(n) & ; b=B \\ \sigma_{b-1}(n)+\sigma_{b}(n)+\sigma_{b+1}(n) & ; \text { else }\end{cases}
$$

The measurement parameters were set like for measurement number 1 from Table 2. For each measurement, the first test signal was presented during at least $15 \mathrm{~s}$. Then, modulation was varied stepwise, as described above, and for each step, the test signal was presented long enough to measure in steady-state. Differential measurements were made, averaging five DFT windows from the last $5 \mathrm{~s}$ of each measurement signal. Attenuation as a function of modulation was extracted from the measurement data, following a procedure that is given in [4], which extracts the effective attenuation of subband number $b$ from the measured frequency responses. The obtained result is shown in Figure 10. The test is passed, because the figure shows that indeed the attenuation as a function of modulation depth is approximately as shown in Figure 1.

\subsubsection{Verification of the superposition with transient noise reduction attenuation}

Transient noise reduction systems target a special kind of noise that has been reported to be one reason for annoyance among hearing instrument users [38,39]: non-speech transient noises, i.e., signals with a fast change of level over time.

Since transient noise reduction systems should act in addition to traditional noise reduction, they are a good example for the superposition of additional attenuation according to requirement 1.3 from Table 1 .

This section proposes a test that addresses the stated requirement. Since the scope of this article is limited to steady-state responses of modulation-based noise reduction subsystems, we will not cover the verification of a

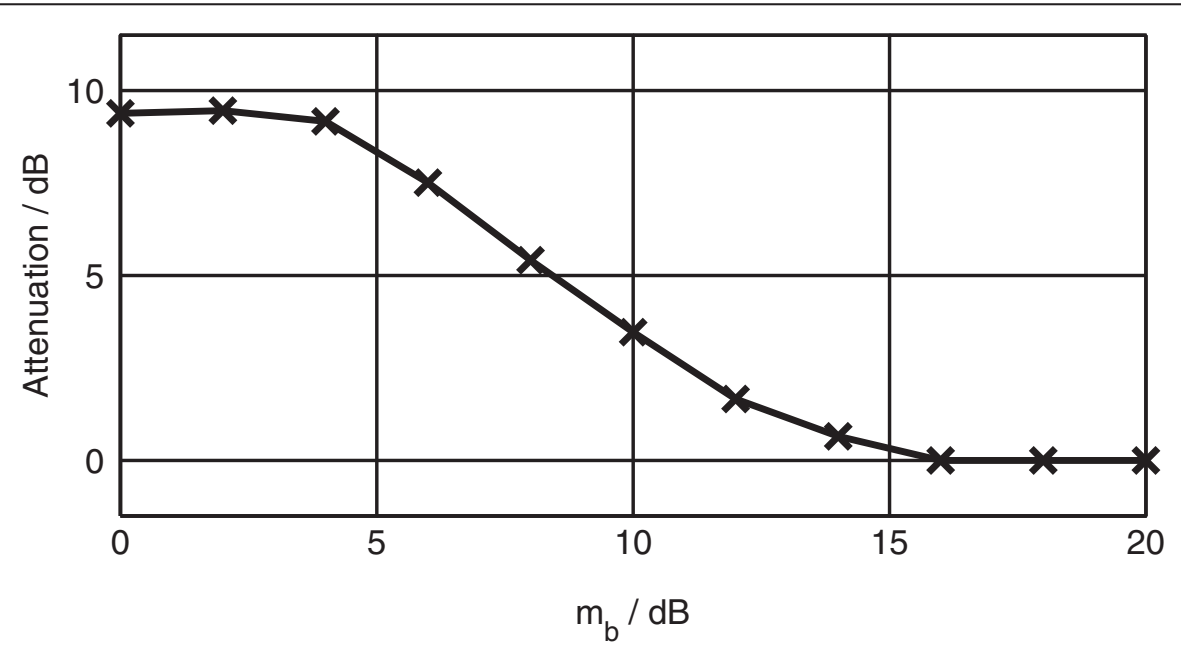

Figure 10 Measured dependency of noise reduction attenuation on modulation depth parameter $m_{b}$. 
transient noise reduction subsystem here. Rather, we show how to test that the traditional noise reduction subsystem has maintained its steady-state performance after a short intervention of a superposed transient noise reduction subsystem.

The challenge here is to insert a transient event into the test stimulus, but still enabling the observation of the frequency response. Although the given test case looks like a good application area for time-frequency approaches like wavelets, we have chosen to stay with the verification techniques that have been presented so far, because we consider the typical mutual exclusion of precise time resolution and precise frequency resolution in time-frequency techniques as a problem for the given test case.

The approach we propose here operates with absolute amplitude measurements in the time before and during a transient noise reduction interaction, and switches to a differential frequency response measurement right afterward. The test signal $s$ is proposed in the equation below.

$$
s(n)= \begin{cases}a \cdot \operatorname{sine}(n)+b \cdot\left[1+\cos \left(2 \pi \frac{f_{m}}{f_{s}} n\right)\right] \cdot p(n) & ; \frac{n}{f_{s}}<T_{1} \\ c \cdot \operatorname{sine}(n) & ; \frac{n}{f_{s}} \in\left[T_{1} ; T_{2}\right] . \\ p(n) & ; \text { else }\end{cases}
$$

Here $\operatorname{sine}(n)=\sin \left(2 \pi \frac{f_{0}}{f_{s}} n\right) ; p$ is a periodically repeated, 1,023 samples binary $\mathrm{m}$-sequence of $70 \mathrm{~dB}$ SPL, $n$ is the sample index, $f_{s}$ is the sampling rate, $f_{0}$ is the frequency of the stimulating sine (here: $f_{0}=1,094$ $\mathrm{Hz}$ ), and $f_{m}$ is the modulation frequency for modulating the background noise, $a$ and $b$ were chosen such that the level of $a \cdot$ sine $(n)$ was $65 \mathrm{~dB}$ SPL and was $15 \mathrm{~dB}$ higher than the level of $b \cdot\left[1+\cos \left(2 \pi \frac{f_{m}}{f_{s}} n\right)\right] \cdot p(n)$. The modulation frequency was set to $f_{m}=4 \mathrm{~Hz}$, as earlier. The coefficient $c$ was chosen such that the corresponding sine signal had a level of $90 \mathrm{~dB}$ SPL. Furthermore, $T_{1}=40.0 \mathrm{~s} ; T_{2}=40.1 \mathrm{~s}$, and the total stimulus duration was $42.1 \mathrm{~s}$.

The stimulus $s$ from Equation 12 uses a sine signal as a basis for stimulating both the noise reduction subsystem as well as the transient noise reduction subsystem: while a steady-state presentation of the sine signal would be sufficient for triggering the noise reduction, the dramatic change of the sine amplitude at time $T_{1}$ is required to trigger the transient noise reduction. One should note that sine-based signals would not necessarily be the optimum stimuli in testing transient noise reduction systems alone. In this case, however, the sine signal was chosen in order to make mainly the behavior of the modulationbased noise reduction subsystem predictable.

A sample hearing instrument with a modulation-based noise reduction subsystem and a transient noise reduction subsystem was used for measurements based on the stimulus $s$ from Equation 12. Differential DFTbased frequency response measurements were performed during the time after $T_{2}$. They were differential in the sense that both noise reduction and transient noise reduction were disabled for the "off" measurement and were enabled for the "on" measurement.

The result of the measurement is shown in Figure 11: The top diagram shows the differential frequency response obtained in the time after $T_{2}$. It shows the steady-state frequency response of the noise reduction subsystem under test as a result of the stimulation with a pure tone of $1,094 \mathrm{~Hz}$. The bottom sub-figure shows the time domain plot of the system under test's output signal (acquired in a non-differential measurement). It captures the transient noise reduction event. It allows the tester to verify if the attenuation by the transient noise reduction was correctly superposed with the present steady-state noise reduction response. The attenuation of the transient noise reduction subsystem is evident from the shape of the signal in the bottom subfigure: while the input signal of the system under test has a sudden change in level at $40 \mathrm{~s}$ according to the above definition of $T_{1}$ in Equation 12, the output signal has a smooth transition between the two levels, as a consequence of the transient noise reduction subsystem's operation.

The tester can verify that the noise reduction subsystem's response in the top subfigure equals the expected steady-state response. The tester can also verify that the envelope of the signal in the bottom subfigure is attenuated compared to cases with an inactive noise reduction subsystem. The additional amount of attenuation shall be exactly the steady-state attenuation of the noise reduction subsystem to pass the test of correct superposition of both subsystems.

\subsection{Discussion}

The verification of isolated requirements like the subbands' crossover frequencies or the superposition of noise reduction effect with effects of other subsystems could successfully be demonstrated. The core requirements about the modulation-based behavior of the noise reduction subsystem, however, lead to measurements exposing side effects or imprecise results, like shown in Figure 9. Therefore, the results shown in that figure need to be discussed further.

We assume that an inadequate test signal rather than a problem in the system under test explains the side effect that has been marked by an arrow in Figure 9b. Our theory is that the side effect occurs due to the side lobe energy according to Figure 3 , which shows the spectrum level of one of the signals $v_{b}$ involved in the measurement according to Figure $9 \mathrm{~b}$. It can easily be 


\section{Differential response of the noise reduction after stimulation with $1094 \mathrm{~Hz}$}
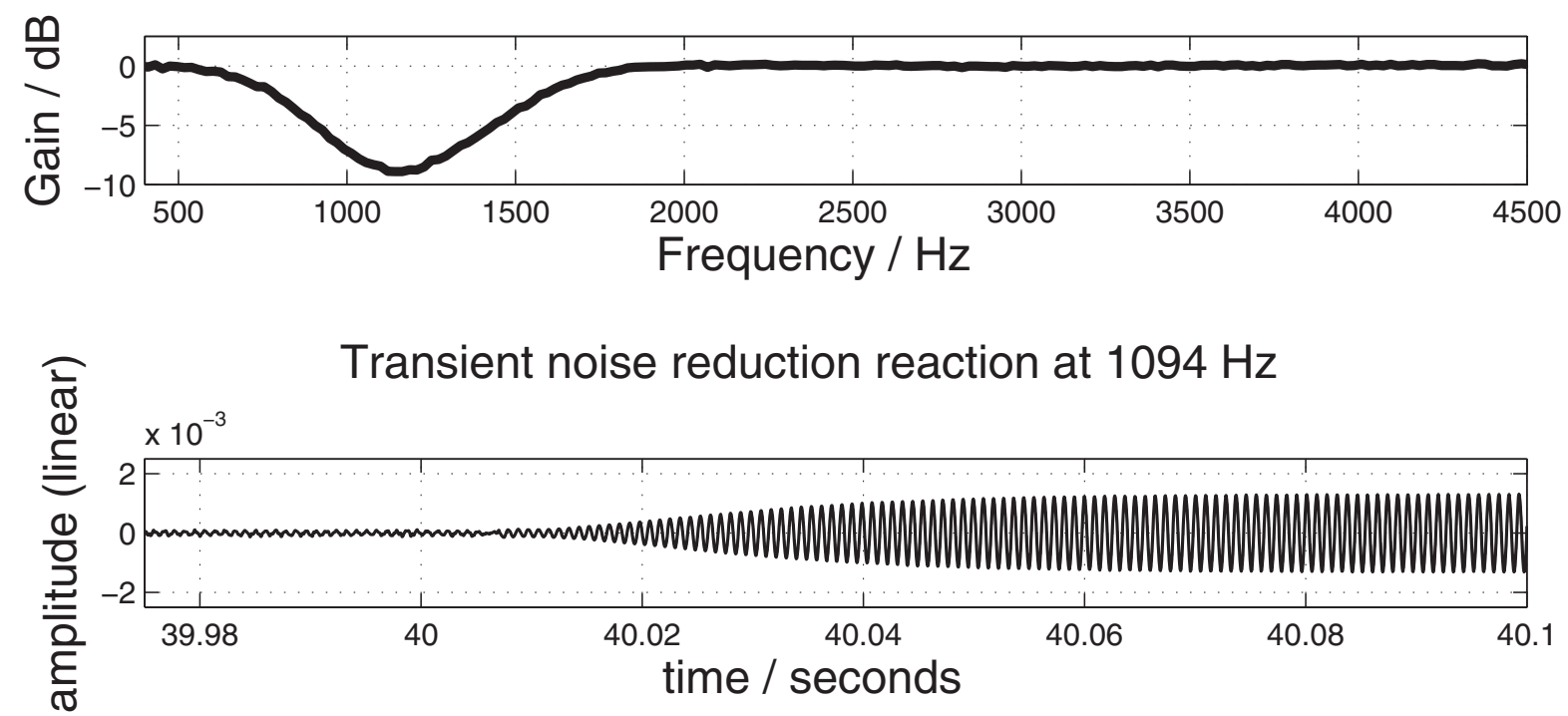

Figure 11 Typical test output in verifying a noise reduction subsystem's interaction with a transient noise reduction subsystem.

seen that the side effect occurs in the same region where the unwanted side lobe in the power spectrum of the DIBS stimulus is present. An explanation for the side effect is that the unmodulated side lobe energy of the stimulus targeted at subband $b$ enters the subband that spans the frequencies at which the side effect can be observed. Since the stimulus targeted at these frequencies is unmodulated, the side-lobe energy can make the effective signal in that subband less modulated: it disturbs the modulation pattern. According to this theory, particularly noise reduction subsystems configured to have a high sensitivity to unmodulated noise would apply attenuation in the subband corresponding to the frequency range of the side lobe energy, which is consistent with the observations reported in Table 3.

Also Figure 9c shows a deviation of the measured frequency response from the expected response: at the upper $3 \mathrm{~dB}$ corner frequency of the subband of interest, the system applies more than $3 \mathrm{~dB}$ attenuation. Our theory for explaining this effect was derived from the spectrogram [40] of the used multi-sine-based measurement signal $\theta_{b}$ with $\forall_{i}: \phi_{i}=0$, as shown in Figure 12 . The darker the plot area, the more energy is present. The black stripe-like patterns in Figure 12 show that the energy in subband $b$ moves up and down in frequency similar to a chirp, where "predominantly one frequency

[is] followed in time by another" [27].

The two white areas in the middle of Figure 12 display a modulation valley; this means a part of the signal in which the cosine in Equation 3 is close to -1. During a modulation valley in subbands other than $b$, the chirplike signal of subband $b$ has most of its energy close to its upper band limit. Our theory is that this disturbs the modulation pattern of subband $b+1$ via non-idealities of band split filters in the noise reduction subsystem under test, and, in consequence, leads to application of undesirable attenuation in subband $b+1$.

In the measurement according to Figure $9 \mathrm{~d}$, the phase shift $\forall_{i \neq b}: \phi_{i}=\pi / 2$ in the modulating cosine has moved the modulation valley to a point in time at which most energy in each subband is present close to the subband's center frequency. This means that, during a modulation valley in most of the subbands, the unmodulated energy of subband $b$ is not dense close to this subband's corner frequencies, where it could have an impact on other subbands. Our theory is that the unmodulated signal in subband $b$ cannot disturb the other subbands' modulation patterns with $\phi_{i}=\pi / 2$, because at points in time at which the energy in subband $b$ can have an impact on other subbands, these now have modulation peaks, which are only affected in a negligible way if additional energy enters the subband. This can explain why the measurement result according to Figure 9d is closest to the expected response of the subsystem under test, as shown in Figure 9a.

\section{Limitations}

In order to scope the application range of the presented work, this section discusses the limitations of both the 


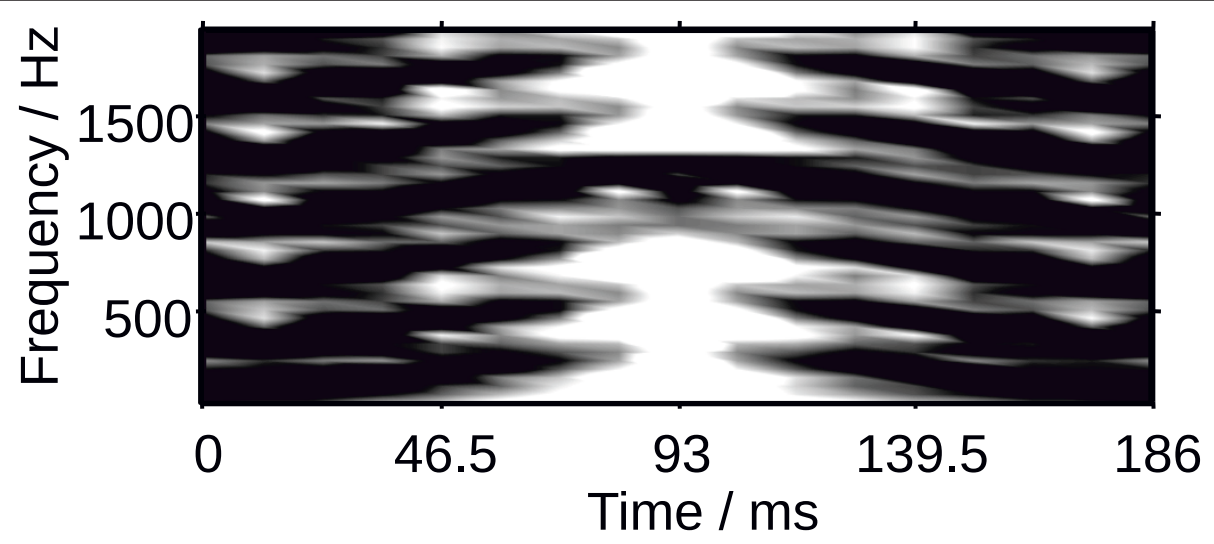

Figure 12 Spectrogram [40] of the test signal that was used for measurement \#2 according to Table 2. Signal power is normalized.

general procedure that has been proposed and the concrete techniques for stimulus-based measurement we demonstrated in the application to noise reduction subsystems of hearing instruments.

\subsection{Limitations of the procedure}

\subsubsection{Limitations of the scope}

The presented procedure targets those kinds of signal processing systems whose processing functionality is based on input signals. This means that systems without signal inputs cannot be verified based on the procedure. This would for example disqualify a system for speech synthesis based on text files as a candidate for applying the procedure.

Since the procedure is based on synthetic stimuli, it cannot assess the performance of the system under test in realistic conditions. Therefore it can only be used in monitoring the technical performance of the system, but cannot be used in determining how well the system fulfills user needs.

The application range of synthesized test signals is in general limited to the one system the stimuli have been targeted at, because the procedure does not address the effect of implementation changes in the system under test on the measurement results obtained with the synthetic stimuli. This means that the validity of test signals has to be re-assessed on product improvements affecting one or more of the subsystems that contribute to the processing of the test signals in the system under test.

\subsubsection{Limitations of the concept}

The procedure is based on the uniformity hypothesis and can therefore only produce valid test results if the partitioning of the system's input domain into equivalence classes holds. Especially for systems with highly non-monotonic input-output relationships we expect difficulty in finding suitable equivalence classes.

The procedure also does not guarantee to find test signals for every given system, because it cannot automatically derive test signals from system specifications, meaning that the equations for signal synthesis have to be invented by the test designer.

\subsection{Limitations of the demonstrated synthesis and measurement techniques}

\subsubsection{Limitations of the scope}

As mentioned in the introduction, the presented procedure cannot replace clinical trials of a noise reduction subsystem in a hearing instrument. Whereas the procedure is generic enough to support various noise reduction algorithms, the exemplarily demonstrated test signals are based on a modulation frequency of 4 or 5.4 $\mathrm{Hz}$, and are thus only suitable for testing noise reduction algorithms with time constants much greater than 250 or $190 \mathrm{~ms}$, respectively. Furthermore, the used measurement procedure assumes a system that is timeinvariant or has at least reached a steady-state condition in which it behaves like a time-invariant system. The exemplarily demonstrated tests are thus inappropriate for time varying systems.

\subsubsection{Limitations of the concept}

Adaptive features other than the one under test may need to be turned off during the measurement, because it is difficult or even impossible to find stimuli that keep all other subsystems in steady-state as a reaction to the stimuli.

Furthermore, the synthesis of test signals is dependent on the design. For example, if crossover frequencies of the demonstrated noise reduction subsystem would change in the course of product innovation, then test signals would need to be re-synthesized, based on the new set of crossover frequencies.

\section{Conclusion and outlook}

We have proposed a procedure for the design of test signals targeted at obtaining test coverage in the measurement-based verification of signal processing systems. 
The procedure is based on identifying requirements toward the system under test and verifying if they have been met. A main goal is test coverage regarding the input parameters of the system under test. Where achieving test coverage is non-trivial, the procedure foresees the separate steps of first describing abstract test sequences in terms of equivalence classes of input parameters to be covered, and secondly synthesizing concrete measurement stimuli to be used with a particular measurement technique.

The procedure has been explored using the stimulusbased verification of modulation-based noise reduction subsystems in hearing instrument as a sample application. All requirements that had been stated for the sample subsystem could be covered with a test.

The comparison of different stimuli showed that some stimuli are more exposed to producing side effects in certain tests than others. For example, it can be concluded from the results that multi-sine synthesis procedures are a good basis for the synthesis of stimuli, if their chirp-like nature is accounted for in test signal design. On the other hand it can be concluded that DIBS-based stimuli can produce side effects if they are used for narrow-band synthesis.

Test signal synthesis was based on the assumption that the noise reduction subsystem under test maintains its steady-state on the one hand during the stimulation with a modulated signal of 4 or $5.4 \mathrm{~Hz}$ modulation frequency and on the other hand after the stimulation with a pure tone that is replaced by a different signal during the actual measurement. This assumption holds for slow noise reduction subsystems, but certainly not for all known noise reduction concepts.

Even though the proposed verification procedure may require new design of stimuli on implementation changes, its abstract way of describing tests provides flexibility with regard to changing assumptions: if the synthesized stimuli or the chosen measurement technique are no longer suited for a given noise reduction technology (e.g., because it does not provide sufficient linearity or time-invariance), then the test design can still be used, and only the choice of stimuli and/or measurement technique has to be reconsidered. For example, NLMS-based measurements can be considered if the system under test is time-variant or too non-linear for the application of the DFT-based measurements we explored here.

\section{Competing interests}

The authors declare that they have no competing interests.

\section{Acknowledgements}

The authors would like to thank Mr. Miquel Sans, Bernafon AG, for contributing his knowledge and some indispensable ideas to the verification techniques related to transient noise reduction systems. They also would like to thank The MathWorks, Inc. for supporting their work on this article.

Received: 30 January 2011 Accepted: 10 November 2011

Published: 10 November 2011

\section{References}

1. I Holube, V Hamacher, M Wesselkamp, Hearing instruments-noise reduction strategies, in Proceedings 18th Danavox Symposium: Auditory Models and Non-linear Hearing Instruments, Kolding, Denmark, pp. 359-377 (1999)

2. V Hamacher, J Chalupper, J Eggers, E Fischer, U Kornagel, H Puder, U Rass, Signal processing in high-end hearing aids: state of the art, challenges, and future trends. EURASIP J Appl Signal Process, 18: 2915-2929 (2005)

3. A Schaub, Digital Hearing Aids (Thieme Medical Publishers, New York, 2008)

4. JG Lamm, AK Berg, CG Glück, Synthetic stimuli for the steady-state verification of modulation-based noise reduction systems. EURASIP J Adv Signal Process. 2009 (2009)

5. JG Lamm, AK Berg, CG Glück, Synthetic signals for verifying noise reduction systems in digital hearing instruments, in EUSIPCO 2008: Proceedings of the 16th European Signal Processing Conference, Lausanne, Switzerland (2008)

6. DJ Richardson, LA Clarke, A partition analysis method to increase program reliability, in Proceedings of the 5th International Conference on Software Engineering, IEEE, San Diego, pp. 244-253 (1981)

7. M Conrad, Systematic testing of embedded automotive software-the classification-tree method for embedded systems (CTM/ES), in Perspectives of Model-Based Testing, ser Dagstuhl Seminar Proceedings, ed. by E Brinksma, W Grieskamp, J Tretmans (Schloss Dagstuhl, Germany, 2005) http://drops. dagstuhl.de/opus/volltexte/2005/325. no 04371 (Dagstuhl, Germany): Internationales Begegnungs- und Forschungszentrum für Infor-matik (IBFI) [Online]

8. M Conrad, Modell-basierter Test eingebetteter Software im Automobil, PhD thesis (TU Berlin, Deutscher Universitätsverlag, 2004)

9. H Blume, M Haller, M Botteck, W Theimer, Perceptual feature based music classification-a DSP perspective for a new type of application. in International Conference on Embedded Computer Systems: Architectures, Modeling, and Simulation, 2008. SAMOS 2008 92-99 (2008)

10. HW Gierlich, New measurement methods for determining the transfer characteristics of telephone terminal equipment, in Proceedings IEEE International Symposium on Circuits and Systems, San Diego, pp. 2069-2072 (1992)

11. AE Hoetink, L Körösssi, WA Dreschler, Classification of steady-state gain reduction produced by amplitude modulation based noise reduction in digital hearing aids. Int J Audiol. 48, 444-455 (2009). doi:10.1080/ 14992020902725539

12. R Bentler, LK Chiou, Digital noise reduction: an overview. Trends Amplif. $10(2), 67-82(2006)$

13. C Antweiler, M Antweiler, System identification with perfect sequences based on the NLMS algorithm. Int J Electron Commun. 49(3), 129-134 (1995)

14. B Widrow, M Hoff, Adaptive switching circuits. in IRE WESCON Convention Record, part 4 96-104 (1960)

15. J Nagumo, A Noda, A learning method for system identification. IEEE Trans Automat Contr. 12(3), 282-287 (1967)

16. HA Barker, RW Davy, System identification using pseudorandom signals and the discrete Fourier transform. Proc IEE. 122(3), 305-311 (1975). doi:10.1049/ piee. 1975.0084

17. ST Nichols, LP Dennis, Estimating frequency response function using periodic signals and the FFT. Electron Lett. 7(22), 662-663 (1971). doi:10.1049/el:19710452

18. L Bougé, N Choquet, L Fribourg, MC Gaudel, Test sets generation from algebraic specifications using logic programming. J Syst Softw. 6, 343-360 (1986). doi:10.1016/0164-1212(86)90004-X

19. AT Bahill, FF Dean, Handbook of Systems Engineering and Management, Wiley, pp. 205-266. ch. 4 Discovering System Requirements) (2009)

20. M Grochtmann, K Grimm, Classification trees for partition testing. Softw Test Verif Reliab. 3(63-82) (1993)

21. MR Schroeder, Synthesis of low peak factor signals and binary sequences with low autocorrelation. IEEE Trans Inf Theory. 16, 85-89 (1970). doi:10.1109/TIT.1970.1054411

22. S Müller, P Massarani, Transfer-function measurements with sweeps. J Audio Eng Soc. 49(6), 443-471 (2001) 
23. A Van den Bos, RG Krol, Synthesis of discrete-interval binary signals with specified Fourier amplitude spectra. Int J Contr. 30(5), 871-884 (1979). doi:10.1080/00207177908922819

24. K-D Paehlike, H Rake, Binary multifrequency signals-synthesis and application, in Proceedings of the 5th IFAC Symposium on Identification and System Parameter Estimation, vol. 1. (Darmstadt, Germany, 1979), pp. 589-596

25. KR Godfrey, AH Tan, HA Barker, B Chong, A survey of readily accessible perturbation signals for system identification in the frequency domain. Contr Eng Pract. 13(11), 1391-1402 (2005). doi:10.1016/j. conengprac.2004.12.012

26. GT Buracas, GM Boynton, Efficient design of event-related fMRI experiments using m-sequences. Neuroimage. 16(3 Part 1), 801-813 (2002)

27. WM Hartmann, J Pumplin, Periodic signals with minimal power fluctuations. J Acoust Soc Am. 90(4), 1986-1999 (1991). doi:10.1121/1.401678

28. E Van der Ouderaa, J Schoukens, J Renneboog, Peak factor minimization using a time-frequency domain swapping algorithm. IEEE Trans Instrum Meas. 37(1), 145-147 (1988). doi:10.1109/19.2684

29. R Pintelon, J Schoukens, System Identification: A Frequency Domain Approach (IEEE Press, New York, 2001)

30. PE Wellstead, Pseudonoise test signals and the Fast Fourier Transform. Electron Lett. 11(10), 202-203 (1975). doi:10.1049/el:19750154

31. L Ljung, System Identification (Prentice Hall, 1999)

32. JD Lamb, D Rees, Digital processing of system responses to pseudorandom binary sequences to obtain frequency response characteristics using the fast fourier transform. in The Use of Digital Computers in Measurement, IEE Conference Publication. 103, 141-146 (1973)

33. Classification Tree Editor (CTE ${ }^{\odot}$ ) (Razorcat Development $\mathrm{GmbH}$, Berlin) http://www.razorcat.com. Accessed Dec 2010

34. J Mischke, Classification Tree Editor for Embedded Systems, ISBN 3-8311-1848-5 (Books on Demand GmbH, 2001)

35. I Kollár, Frequency domain system identification toolbox V3.3 for Matlab (Gamax Ltd, Budapest, 2004)

36. J Lamm, C Glück, Wey R, Effiziente Verifikation mit hörbarem Erfolg. Swiss Eng stz. 04/2010, 22-23 (2010)

37. IS Bendat, AG Piersol, Random Data-Analysis and Measurement Procedures (Wiley, 2000)

38. J Chalupper, TA Powers, New algorithm is designed to take the annoyance out of transient noise. Hear J. 60(7), 42,44-46,48 (2007)

39. G Keidser, A O'Brien, M Latzel, E Convery, Evaluation of a noise-reduction algorithm that targets non-speech transient sounds. Hear J. 60(2,29,32,34,38-39) (2007)

40. AV Oppenheim, Speech spectrograms using the fast Fourier transform. IEEE Spectr. 7(8), 57-62 (1970)

doi:10.1186/1687-6180-2011-100

Cite this article as: Lamm et al:: Procedure for the steady-state verification of modulation-based noise reduction systems in hearing instruments. EURASIP Journal on Advances in Signal Processing 2011 2011:100.

\section{Submit your manuscript to a SpringerOpen ${ }^{\circ}$ journal and benefit from:}

- Convenient online submission

- Rigorous peer review

- Immediate publication on acceptance

- Open access: articles freely available online

- High visibility within the field

- Retaining the copyright to your article

Submit your next manuscript at $\gg$ springeropen.com 